\title{
CelR-mediated activation of the cellobiose- utilization gene cluster in Streptococcus pneumoniae
}

\author{
Sulman Shafeeq, Tomas G. Kloosterman and Oscar P. Kuipers
}

Correspondence

Oscar P. Kuipers

o.p.kuipers@rug.nl

Received 17 May 2011

Revised 11 July 2011

Accepted 18 July 2011
Department of Molecular Genetics, Groningen Biomolecular Sciences and Biotechnology Institute, University of Groningen, Nijenborgh 7, 9747 AG Groningen, The Netherlands

\begin{abstract}
The human pathogen Streptococcus pneumoniae harbours many genes encoding phosphotransferase systems and sugar ABC (ATP-binding cassette) transporters, including systems for the utilization of the $\beta$-glucoside sugar cellobiose. In this study, we show that the transcriptional regulator CelR, which has previously been found to be important for pneumococcal virulence, activates the expression of the cellobiose-utilization gene cluster (cel locus) of S. pneumoniae. Expression directed by the two promoters present in the cel locus was increased in the presence of cellobiose as sole carbon source in the medium, while expression decreased in the presence of glucose in the medium. Furthermore, we have predicted a 22 bp putative CelR regulatory site (5'-YTTTCCWTAWCAWTWAGGAAAA-3') in the promoters of celA and celB, and in silico analysis showed that it is highly conserved in other pathogenic streptococci as well. Promoter truncations of $c e / A$ and $c e / B$, where the half or full CelR regulatory site was deleted, confirmed that the CelR-binding site in PcelA and PcelB is functional. Transcriptome studies with the celR mutant and in silico prediction of the CeIR regulatory site in the entire D39 genome sequence show that the cel locus is the only cluster of genes under the direct control of CelR. Therefore, CelR is a regulator dedicated to the cellobiose-dependent transcriptional activation of the cel locus.
\end{abstract}

\section{INTRODUCTION}

The major Gram-positive human pathogen Streptococcus pneumoniae has the ability to colonize the nasopharynx (Bogaert et al., 2004) and from there to spread to different parts of the human body, causing serious infections like pneumonia, meningitis, otitis media and sepsis (Kadioglu et al., 2008; Obaro \& Adegbola, 2002). For S. pneumoniae, a big challenge for survival in the host environment is adaptation to the changing nutritional conditions encountered in the different niches inside the host, according to which it has to tune the expression of the appropriate genes (Brown et al., 2008; Mitchell, 2003; Shelburne et al., 2008).

Glucose is the preferred source of energy for many bacteria, but the presence of different sugar-specific phosphotransferase systems (PTSs) gives them the ability to utilize other carbon sources as well (Deutscher et al.,

Abbreviations: CRE, catabolite-responsive element; PTS, phosphotransferase system.

The microarray data reported have been deposited with the Gene Expression Omnibus (GEO) with the accession number GSE30891.

A supplementary table, listing PCR primers used in this study, is available with the online version of this paper.
2006). Bacteria are able to ferment different $\beta$-glucosides such as cellobiose, aesculin, arbutin and salicin, which are commonly present in plants (Kiliç et al., 2004). Cellobiose can be a potential source of energy for different streptococci as well (Keyhani \& Roseman, 1997; McKessar \& Hakenbeck, 2007; Zeng \& Burne, 2009). In S. pneumoniae, the gene cluster for cellobiose utilization ( $\mathrm{cel}$ locus) comprises seven genes, encoding a 6-phospho$\beta$-glucosidase (CelA), a putative DNA-binding transcriptional regulator (CelR), the $\mathrm{A}, \mathrm{B}$ and $\mathrm{C}$ domains of a cellobiose PTS EII ${ }^{\mathrm{Cel}}$ (CelB, CelC and CelD), and two hypothetical proteins. It has been shown that $S$. pneumoniae lacking $c e l R, c e l D$ or the hypothetical gene spr0281 (SPD_0282) was hardly able to grow in a chemically defined medium (CDM) supplemented with cellobiose as a sole carbon source (McKessar \& Hakenbeck, 2007). A similar study has been performed in Streptococcus mutans (Zeng \& Burne, 2009), where strains with deletion of celA, $c e l B, c e l C, c e l D$ or $c e l R$ were unable to grow in medium with cellobiose as a sole source of energy.

Interestingly, the extracellular matrix (ECM) of mammalian tissues is rich in glycosaminoglycans that contain repeating units of $\beta$-linked disaccharides (Kiliç et al., 2004). The degradation of glycosaminoglycans from the mammalian ECM may release structural analogues of cellobiose 
(Kiliç et al., 2004). Although it is unlikely that $S$. pneumoniae encounters cellobiose in the human body, it is possible that it can utilize structurally similar $\beta$ glucosides that are derived by degradation of the mammalian ECM (Kiliç et al., 2004; King, 2010).

The two-component system TC08 is one of the 13 twocomponent systems (Throup et al., 2000) found in S. pneumoniae and has been shown to be involved in the regulation of the pilus locus in strain TIGR4 (Song et al., 2009). In strain R6, it regulates the cel locus: a mutant producing a hyperactive variant of HK08 displayed high repression of transcription of the cel genes (McKessar \& Hakenbeck, 2007). Moreover, this hk08 mutant was not able to grow in the presence of cellobiose as sole energy source (McKessar \& Hakenbeck, 2007). On the other hand, in $S$. mutans the cel locus was activated by the transcriptional regulator CelR and a strain lacking $c e l R$ was unable to grow in medium with cellobiose as sole energy source (Zeng \& Burne, 2009). Furthermore, the celA gene in the cel locus encodes one of the three $\beta$-glucosidases present in S. mutans, and a strain in which celA was deleted was unable to ferment cellobiose, amygdalin, gentiobiose and salicin (Old et al., 2006). Deletion of the other two $\beta$-glucosidases in S. mutans had no effect on the fermentation of these four $\beta$-glucosides (Old et al., 2006). These studies demonstrate the importance of CelA in the fermentation of cellobiose and the role of CelR as an activator of the cel locus in S. mutans.

In this study, we show that CelR acts as a cellobiosedependent transcriptional activator of the cel locus in $S$. pneumoniae as well. Furthermore, in the presence of cellobiose or glucose, the expression of the cel locus was not affected by deletion of either $h k 08$ or $\operatorname{ccp} A$. Investigation of the $c e l R$ deletion strain by means of DNA microarray analyses demonstrated that, besides the cel locus, CelR has no other targets under the conditions tested. By MEME motif search, a putative regulatory site of CelR was identified in the promoter regions of celA and $c e l B$, which is conserved in $\mathrm{P} c e l A$ and $\mathrm{P} c e l B$ of other streptococci as well. The functionality of this CelR regulatory site was proven by means of promoter truncation, showing that deletion of the half or full regulatory sequence leads to total inactivation of the $\mathrm{celA}$ and $c e l B$ promoters.

\section{METHODS}

Bacterial strains and growth conditions. Bacterial strains and plasmids used in this study are listed in Table 1. M17 broth (Terzaghi \& Sandine, 1975) supplemented with $0.5 \%(\mathrm{w} / \mathrm{v})$ glucose was used for growing S. pneumoniae D39 wild-type (Lanie et al., 2007) on blood agar plates supplemented with $1 \%(\mathrm{v} / \mathrm{v})$ defibrinated sheep blood in micro-aerophilic conditions at $37^{\circ} \mathrm{C}$. For $\beta$-galactosidase assays, derivatives of $S$. pneumoniae D39 were grown in M17 medium supplemented with different sugars (glucose or cellobiose at the concentrations described in Results), and cells were harvested at $\mathrm{OD}_{600}$ 0.3. For selection on antibiotics, media were supplemented
Table 1. Strains and plasmids used in this study

\begin{tabular}{|c|c|c|}
\hline Strain/plasmid & Description & Source \\
\hline \multicolumn{3}{|l|}{ S. pneumoniae } \\
\hline D39 & Serotype 2 strain, cps 2 & $\begin{array}{c}\text { Lanie et al. } \\
(2007)\end{array}$ \\
\hline$\Delta c e l R$ & D39 $\Delta c e l R::$ Spec $^{\mathrm{R}}$ & This study \\
\hline$\Delta c c p A$ & D39 $\Delta c c p A::$ Spec $^{\mathrm{R}}$ & $\begin{array}{l}\text { S. M. } \\
\text { Carvalho }\end{array}$ \\
\hline$\Delta h k 08$ & D39 $\Delta h k 08::$ Ery $^{\mathrm{R}}$ & This study \\
\hline SS401 & D39 $\Delta$ bgaA:: PcelA-lacZ; Tet $^{\mathrm{R}}$ & This study \\
\hline SS402 & D39 $\Delta$ bgaA::Pspd_0278-lacZ; Tet $^{\mathrm{R}}$ & This study \\
\hline SS403 & D39 $\Delta$ bgaA : : PcelB-lacZ; Tet $^{\mathrm{R}}$ & This study \\
\hline SS404 & D39 $\Delta$ bgaA : : PcelR-lacZ; Tet $^{\mathrm{R}}$ & This study \\
\hline SS405 & D39 $\Delta$ bgaA : : PcelD-lacZ; Tet $^{\mathrm{R}}$ & This study \\
\hline SS406 & $\Delta c e l R \Delta b g a A:: \mathrm{P} c e l A-l a c Z ;$ Tet $^{\mathrm{R}}$ & This study \\
\hline SS407 & $\Delta c e l R \Delta$ bgaA:: PcelB-lacZ; Tet $^{\mathrm{R}}$ & This study \\
\hline SS408 & $\Delta c c p A \Delta b g a A:: \mathrm{P} c e l A-l a c Z ;$ Tet $^{\mathrm{R}}$ & This study \\
\hline SS409 & $\Delta c c p A \Delta b g a A:: \mathrm{P} c e l B-l a c Z ; \mathrm{Tet}^{\mathrm{R}}$ & This study \\
\hline SS410 & $\Delta h k 08 \Delta$ bgaA:: PcelA-lacZ; Tet $^{\mathrm{R}}$ & This study \\
\hline SS411 & $\Delta h k 08 \Delta b g a A::$ PcelB-lacZ; Tet $^{\mathrm{R}}$ & This study \\
\hline SS412 & D39 $\Delta$ bgaA:: PcelA F-lacZ; Tet $^{\mathrm{R}}$ & This study \\
\hline SS413 & D39 $\Delta$ bgaA :: PcelA H-lacZ; Tet ${ }^{\mathrm{R}}$ & This study \\
\hline SS414 & D39 $\Delta$ bgaA : : PcelA N-lacZ; Tet $^{\mathrm{R}}$ & This study \\
\hline SS415 & D39 $\Delta$ bgaA:: PcelB F-lacZ; Tet $^{\mathrm{R}}$ & This study \\
\hline SS416 & D39 $\Delta$ bgaA : : PcelB H-lacZ; Tet $^{\mathrm{R}}$ & This study \\
\hline SS417 & D39 $\Delta$ bgaA:: PcelB N-lacZ; Tet $^{\mathrm{R}}$ & This study \\
\hline \multicolumn{3}{|l|}{ E. coli } \\
\hline EC1000 & $\begin{array}{l}\mathrm{Km}^{\mathrm{R}} \text {; MC1000 derivative carrying } \\
\text { a single copy of the pWV1 repA } \\
\text { gene in } \operatorname{glg} B\end{array}$ & $\begin{array}{r}\text { Laboratory } \\
\text { collection }\end{array}$ \\
\hline \multicolumn{3}{|l|}{ Plasmids } \\
\hline pPP2 & $\begin{array}{l}\mathrm{Amp}^{\mathrm{R}} \mathrm{Tet}^{\mathrm{R}} \text {; promoterless lac } Z \text {, } \\
\text { for replacement of bgaA with } \\
\text { promoter-lacZ fusion; deriva- } \\
\text { tive of pTP1 }\end{array}$ & $\begin{array}{l}\text { Halfmann } \\
\text { et al. } \\
(2007)\end{array}$ \\
\hline pSS401 & pPP2 PcelA & This study \\
\hline pSS402 & pPP2 PSPD_0278 & This study \\
\hline pSS403 & pPP2 PcelB & This study \\
\hline pSS404 & $\mathrm{pPP} 2 \mathrm{Pcel}$ & This study \\
\hline pSS405 & pPP2 PcelD & This study \\
\hline pSS406 & pPP2 PcelA $F$ & This study \\
\hline pSS407 & pPP2 PcelA $H$ & This study \\
\hline pSS408 & pPP2 PcelB N & This study \\
\hline pSS409 & pPP2 PcelB F & This study \\
\hline pSS410 & pPP2 PcelB $H$ & This study \\
\hline pSS411 & pPP2 PcelB N & This study \\
\hline
\end{tabular}

with the following concentrations of antibiotics: erythromycin, $0.25 \mu \mathrm{g} \mathrm{ml}^{-1}$ for S. pneumoniae and $120 \mu \mathrm{g} \mathrm{ml}^{-1}$ for Escherichia coli; spectinomycin, $150 \mu \mathrm{g} \mathrm{ml}{ }^{-1}$; tetracycline, $2.5 \mu \mathrm{g} \mathrm{ml}^{-1}$ for $S$. pneumoniae; and ampicillin, $100 \mu \mathrm{g} \mathrm{ml}^{-1}$ for E. coli. All bacterial strains used in this study were stored in $10 \%(\mathrm{v} / \mathrm{v})$ glycerol at $-80{ }^{\circ} \mathrm{C}$.

DNA isolation and manipulation. All DNA manipulations in this study were done as described previously (Kloosterman et al., 2006a). For PCR amplification, chromosomal DNA of S. pneumoniae D39 wild-type (Lanie et al., 2007) was used. Primers used in this study are listed in Supplementary Table S1, available with the online version of 
this paper, and based on the sequence of the D39 genome (Lanie et al., 2007).

Construction of deletion mutants of hk08 and celR. $h k 08$ and $c e l R$ deletion mutants were made by allelic replacement with an erythromycin- and a spectinomycin-resistance marker, respectively. Briefly, primers HK08-1/HK08-2, HK08-3/HK08-4, SPD0280-1/ SPD0280-2 and SPD0280-3/SPD0280-4 were used to generate PCR fragments of the left and right flanking regions of $h k 08$ and $c e l R$, respectively. PCR products of the erythromycin- and spectinomycinresistance markers were generated with primers Ery-F/Ery- $\mathrm{R}$ and Spec-F/Spec-RP-New from plasmids pORI28 and pORI38 (Leenhouts et al., 1996), respectively. Then, by means of overlap extension PCR, the left and right flanking regions of $h k 08$ and $c e l R$ were fused to these resistance genes. The resulting PCR products were transformed to $S$. pneumoniae D39 wild-type and selection of the mutant strains was done on the appropriate antibiotic. Erythromycin- and spectinomycin-resistant clones were further examined for the presence of the $\Delta h k 08$ and $\Delta c e l R$ deletion by PCR.

Construction of lac $Z$ fusions and $\boldsymbol{\beta}$-galactosidase assays. Chromosomal transcriptional lacZ fusions to the celA, celB, celR, celD and SPD_0278 promoters were constructed in the integration plasmid pPP2 (Halfmann et al., 2007) via double crossover in the bgaA gene with primer pairs listed in Supplementary Table S1, leading to plasmids pSS401-405. These lac $Z$ fusion constructs were introduced into D39 wild-type cells, resulting in strains SS401-405. PcelA-lacZ and PcelB-lacZ were also transformed to the celR, ccpA and $h k 08$ deletion strains, resulting in SS406-411. All plasmid constructs were checked by sequencing.

Specific $\beta$-galactosidase activity was measured as described previously (Kloosterman et al., 2006a) using cells grown in M17 with appropriate sugars (exact concentrations are given in Results) which were harvested in the mid-exponential phase of growth.

Construction of celA and celB promoter subclones in pPP2. The following promoter subclones of the $c e l A$ and $c e l B$ promoters were made in pPP2 (primer pairs are listed in Supplementary Table S1): PcelA-F (truncated just a few bases upstream of the CelR regulatory site), PcelA-H (with the deletion of half of the CelR regulatory site), $\mathrm{P} c e l A-N$ (full CelR regulatory site deleted), $\mathrm{P} c e l B-F$ (truncated just a few bases upstream of CelR regulatory site), $\mathrm{P} c e l B-H$ (with the deletion of half of the CelR regulatory site) and PcelB-N (full CelR regulatory site deleted), resulting in plasmids pSS406-411. These lac $Z$ fusion constructs were introduced into D39 wild-type, resulting in strains SS412-417.

Microarray analysis. For transcriptome analysis the $S$. pneumoniae D39 wild-type strain and its isogenic celR mutant were grown in three biological replicates in CM17 (M17 $+0.5 \%$ cellobiose) medium and harvested in the mid-exponential phase. All other procedures regarding the DNA microarray experiment were performed as described previously (Kloosterman et al., 2006b; Shafeeq et al., 2011b).

Microarray data analysis. DNA microarray data were analysed as described previously (Kloosterman et al., 2006b; Shafeeq et al., 2011a). In brief, DNA microarray slides were scanned with a Genepix 4200 laser scanner at $10 \mu \mathrm{m}$ resolution. Array Pro 4.5 (Media Cybernetics) was used to analyse the slides. The MicroPrep software package (van Hijum et al., 2003) was used to obtain and analyse the microarray data. The expression ratio of the celR mutant over the D39 strain was calculated from the measurements of at least seven spots by Cyber-T (Long et al., 2001). For identification of differentially expressed genes, only genes with a Bayesian $P$-value $<0.001$ and fold change cut-off of 3 were included. The microarray data have been submitted to GEO under accession number GSE30891.

\section{RESULTS}

\section{Organization of the cellobiose operon in S. pneumoniae D39}

The cel locus of S. pneumoniae D39 wild-type consists of seven genes (SPD_0277-0283), which are organized in two putative transcriptional units starting from $\mathrm{P} c e l A$ and $\mathrm{P} c e l B$ (Fig. 1). The first transcriptional unit comprises two genes, encoding 6-phospho- $\beta$-glucosidase (CelA) and a hypothetical protein (SPD_0278), respectively. The second unit encodes the predicted cellobiose-specific PTS components IIBAC (CelB, CelC and CelD), a DNA-binding transcriptional regulator (CelR) and a hypothetical transmembrane protein (SPD_0282).

To prove that the cel locus is transcribed in two transcriptional units, transcriptional lac $Z$ fusions to all intergenic regions in this locus were constructed in plasmid pPP2 and introduced into D39 wild-type. $\beta$-Galactosidase assays were performed with the strains containing these lacZ fusions grown in $\mathrm{CM} 17$ (M17 $+0.5 \%$ cellobiose). $\mathrm{P} c e l A$ and $\mathrm{PcelB}$ were highly active in the presence of cellobiose, while no expression from the other intergenic regions was observed (Table 2). These data demonstrate that the cel locus is organized in two transcriptional units originating from the promoters of $c e l A$ and $c e l B$.

\section{Cellobiose induces and glucose nullifies the expression of the cel locus}

To investigate the transcriptional response of the cel locus of $S$. pneumoniae to different concentrations of cellobiose and glucose,wild-type strain D39 containing PcelA-lacZ or $\mathrm{P} c e l B-l a c Z$ was grown in M17 with different concentrations of these sugars and $\beta$-galactosidase assays were performed with these strains. The data revealed that increasing concentrations of cellobiose led to increased expression of both promoters, whereas increasing the concentration of glucose led to decreased expression of both promoters (Table 3). To study in more detail the role of cellobiose and glucose in the regulation of the $\mathrm{cel}$ locus, $\beta$-galactosidase activity was measured in strain D39 containing PcelA-lacZ and $\mathrm{P} c e l B-l a c Z$ at a constant concentration of glucose, and with increasing concentrations of cellobiose. Even with a five times higher concentration of cellobiose than glucose, the latter prevents transcription from PcelA and PcelB. These results show that cellobiose activates the expression

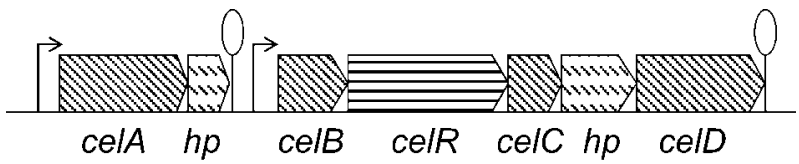

Fig. 1. Organization of the cel locus in S. pneumoniae strain D39. Lollipops and bent arrows indicate putative terminators and putative promoters, respectively. 
Table 2. Specific $\beta$-galactosidase activity of D39 wild-type containing different transcriptional fusions grown in $\mathrm{CM} 17$ medium

The PcelA-lacZ, PcelB-lacZ, PcelD-lacZ, PcelR-lacZ and PSPD_0278lac $Z$ transcriptional fusions were grown in $\mathrm{CM} 17$ (M17+0.5\% cellobiose) medium. Standard deviations of three independent measurements are given in parentheses.

\begin{tabular}{|lc|}
\hline Promoter & $\begin{array}{c}\text { Specific } \boldsymbol{\beta} \text {-galactosidase } \\
\text { activity (Miller units) }\end{array}$ \\
\hline celA & $805(17)$ \\
celB & $143(4.0)$ \\
celD & $8(1.1)$ \\
SPD_0278 & $5(1.0)$ \\
& $6(0.3)$ \\
\hline
\end{tabular}

of the cel locus, while glucose decreases its expression (Table 3).

\section{Role of HK08 and CcpA in the expression of the cel operon}

In a previous study (McKessar \& Hakenbeck, 2007) it was observed that TCS08 is involved in the transcriptional regulation of the cel locus in S. pneumoniae R6. Therefore, to elucidate the putative role of HK08 in the sugardependent transcription of the cel locus in D39, expression of the PcelA-lacZ and PcelB-lacZ transcriptional fusions was measured in an $h k 08$ deletion strain that was created by allelic replacement with an erythromycin-resistance marker. Interestingly, despite deletion of $h k 08, \mathrm{P} c e l A$ and $\mathrm{P} c e l B$ were, in the presence of cellobiose, still expressed to a similar extent as in D39 wild-type, and hardly expressed in the presence of glucose (Table 4). This shows that the

Table 3. Specific $\beta$-galactosidase activity of D39 wild-type containing the PcelA-lacZ or PcelB-lacZ transcriptional fusions grown in M17 medium with different concentrations of cellobiose (C) and/or glucose (G)

Standard deviations of three independent measurements are given in parentheses.

\begin{tabular}{|c|c|c|}
\hline \multirow[t]{2}{*}{ Medium } & \multicolumn{2}{|c|}{$\begin{array}{l}\text { Specific } \beta \text {-galactosidase } \\
\text { activity (Miller units) }\end{array}$} \\
\hline & PcelA & PcelB \\
\hline M17 & $80(3.0)$ & $56(5.0)$ \\
\hline $\mathrm{M} 17+0.2 \% \mathrm{G}$ & $8(1.0)$ & $6(0.5)$ \\
\hline $\mathrm{M} 17+0.2 \% \mathrm{C}$ & $452(21)$ & $98(4.0)$ \\
\hline $\mathrm{M} 17+0.5 \% \mathrm{G}$ & $7(1.0)$ & $6(0.6)$ \\
\hline $\mathrm{M} 17+0.5 \% \mathrm{C}$ & $770(54)$ & $140(4.0)$ \\
\hline $\mathrm{M} 17+0.1 \% \mathrm{G}+0.1 \% \mathrm{C}$ & $9(0.9)$ & $7(1)$ \\
\hline $\mathrm{M} 17+0.1 \% \mathrm{G}+0.2 \% \mathrm{C}$ & $11(1.2)$ & $9(0.8)$ \\
\hline $\mathrm{M} 17+0.1 \% \mathrm{G}+0.5 \% \mathrm{C}$ & $10(1.1)$ & $8(1)$ \\
\hline
\end{tabular}

Table 4. Specific $\beta$-galactosidase activity of D39 wild-type and the $\Delta c e / R, \triangle h k 08$ and $\triangle c c p A$ mutants containing the PcelA-lacZ or PcelB-lacZ transcriptional fusions grown in M1 7 medium plus $0.5 \%$ glucose or cellobiose

GM17, M17 + 0.5\% glucose; CM17, M17 $+0.5 \%$ cellobiose. Standard deviations of three independent measurements are given in parentheses.

\begin{tabular}{|lrrrr|}
\hline \multirow{2}{*}{ Promoter } & \multicolumn{4}{c|}{ Specific $\boldsymbol{\beta}$-galactosidase activity (Miller units) } \\
\cline { 2 - 5 } & \multicolumn{1}{c}{ WT } & \multicolumn{1}{c}{$\boldsymbol{\Delta h \boldsymbol { k } 0 \boldsymbol { 8 }}$} & $\boldsymbol{\Delta} \boldsymbol{\Delta c e l \boldsymbol { R }}$ & \multicolumn{1}{c|}{$\boldsymbol{\Delta c \boldsymbol { c } \boldsymbol { A }}$} \\
\hline PcelA (GM17) & $7.5(0.5)$ & $6(0.1)$ & $6(0.2)$ & $5(0.4)$ \\
PcelA (CM17) & $791(27)$ & $801(33)$ & $5(0.1)$ & $779(35)$ \\
PcelB (GM17) & $8(0.2)$ & $7(0.4)$ & $4(0.7)$ & $5(0.2)$ \\
PcelB (CM17) & $133(4.0)$ & $128(7.0)$ & $5(0.5)$ & $121(2.0)$ \\
\hline
\end{tabular}

observed carbon-source-dependent regulation does not go via TCS08, but that TCS08 probably regulates the cel locus via an as yet unidentified signal.

CcpA is responsible for the main part of carbon catabolite regulation in Gram-positive bacteria including S. pneumoniae. CcpA mediates its repression by binding to cataboliteresponsive element (CRE) sites present in its target promoters (Lulko et al., 2007; Zomer et al., 2007). To determine the putative role of CcpA in the regulation of the cel locus, $\mathrm{P} c e l \mathrm{~A}$ and $\mathrm{P} c e l B$ were analysed for the presence of a putative CRE site. Interestingly, PcelA contains a putative CRE box (5'-AAGAATGCGTTTTCAT-3'). However, in the presence of glucose or cellobiose we could not demonstrate an effect of CcpA on the expression of PcelA and PcelB (Table 4). Therefore, it is likely that CcpA has, as in $S$. mutans (Zeng \& Burne, 2009), no role in the regulation of the cel locus in S. pneumoniae and that the CRE box present in PcelA is not functional, which is in agreement with a recent study (S. M. Carvalho and others, unpublished).

\section{CelR acts as a transcriptional activator of the cel locus in the presence of cellobiose in S. pneumoniae}

As the $\operatorname{ccp} A$ and $h k 08$ mutations have no effect on the regulation of the $\mathrm{cel}$ locus by cellobiose and glucose, we investigated the role of $c e l R$, the second gene of transcriptional unit 2 of the cel locus, which encodes a putative DNA binding transcriptional regulator. To find the effect of its mutation on the expression of the cel locus, PcelA-lacZ and PcelB-lacZ transcriptional fusions were introduced into a $\Delta c e l R$ strain. Both promoters completely lost their activity even in the presence of cellobiose (Table 4). These data suggest that celR acts as a transcriptional activator of the cel locus in the presence of cellobiose.

\section{DNA microarray analysis of $\Delta c e / R$}

To investigate the effect of the celR deletion on the transcriptome of $S$. pneumoniae, D39 wild-type was 
compared with its isogenic celR deletion mutant grown in CM17 (M17+0.5\% cellobiose) medium. As the celR mutant grows more slowly than the wild-type in CM17 medium, cells were harvested in the mid-exponential growth phase according to their growth rate. Table 5 summarizes the results of the transcriptome changes induced by the deletion of $c e l R$. There was no broad effect of $c e l R$ deletion on the transcriptome of $S$. pneumoniae D39, since after applying the criteria of at least a threefold difference as the threshold change and a $P$-value $<0.001$, the cel locus was the only cluster of genes that was significantly downregulated in the $c e l R$ deletion strain and no other big responses were observed in the transcriptome. This suggests that the cel locus is the only target of CelR that is activated in the presence of cellobiose.

\section{Prediction of a celR regulatory site in PcelA and PcelB}

By applying a MEME motif sampler search (Bailey \& Elkan, 1994) a 22 bp palindromic sequence (5'-YTTTCCWTAWCAWTWAGGAAAA-3') located just upstream of celA and $c e l B$ was found in the promoters of $c e l A$ and $c e l B$ of $S$. pneumoniae D39 wild-type (Fig. 2b). This stretch of DNA might serve as a CelR regulatory site in S. pneumoniae. The site is conserved in other sequenced strains of $S$. pneumoniae that possess the cel locus. S. mutans, Streptococcus gordonii, Streptococcus dysgalactiae, Streptococcus gallolyticus, Streptococcus pyogenes and Streptococcus suis contain a cel locus with a similar composition as in S. pneumoniae as well. To find out whether the CelR regulatory site is also conserved in these streptococci the celA and $c e l B$ promoters of these species were analysed for the presence of the putative CelR regulatory sequence. This showed that the CelR regulatory sequence is highly conserved in these streptococci (Fig. 2b). However, a difference in the spacing of a single base pair in

Table 5. Summary of transcriptome comparison of $S$. pneumoniae D39 $\Delta$ celR and D39 wild-type grown in CM17 $(\mathrm{M} 17+0.5 \%$ cellobiose $)$

\begin{tabular}{|c|c|c|}
\hline D39 locus tag & Function & Ratio $^{*}$ \\
\hline SPD_0277 & 6-Phospho- $\beta$-glucosidase, CelA & -81.2 \\
\hline SPD_0278 & Hypothetical protein & -2.4 \\
\hline SPD_0279 & $\begin{array}{l}\text { Cellobiose phosphotransferase } \\
\text { system IIB component, CelB }\end{array}$ & -22.5 \\
\hline SPD_0280 & $\begin{array}{l}\text { DNA-binding transcriptional } \\
\text { regulator, CelR }\end{array}$ & -13.0 \\
\hline SPD_0281 & $\begin{array}{l}\text { Cellobiose phosphotransferase } \\
\text { system IIA component, CelC }\end{array}$ & -22.8 \\
\hline SPD_0282 & Hypothetical protein & -14.1 \\
\hline SPD_0283 & $\begin{array}{l}\text { Cellobiose phosphotransferase } \\
\text { system IIC component, CelD }\end{array}$ & -18.8 \\
\hline
\end{tabular}

${ }^{*}$ Ratios $>3.0$ or $>-3.0$ (signal intensity of D39 $\Delta c e l R$ divided by that of D39 wild-type). SPD_0278 was also included, since it is in the same transcriptional unit. $P$-values all $<0.001$. the middle of the regulatory site was present in S. mutans and $S$. dysgalactiae. The predicted CelR regulatory sequences in the celA and celB promoters of S. mutans, S. gordonii, S. dysgalactiae, S. gallolyticus, S. pyogenes and S. suis were aligned with that of $S$. pneumoniae. In this way, a weight matrix of a $23 \mathrm{bp}$ putative CelR regulatory site (5'-WTTTCCKTWKNCRATAMGGAAAA-3') was created (Fig. 2a).

To investigate if there are more putative CelR targets in the D39 genome, a genome-wide search with the pneumococcal CelR regulatory site was performed. No other stretch of DNA resembling the CelR regulatory site was found, supporting the observation in the microarray analysis with the $c e l R$ mutant that the $c e l$ locus is the only target of CelR.

\section{Verification of the CelR regulatory site in PcelA and PcelB}

To prove the functionality of the identified CelR regulatory sequence in the regulation of the cel locus by CelR, transcriptional lacZ fusions to $5^{\prime}$ truncations of PcelA and PcelB were constructed (Fig. 3). No expression of either of the promoters (PcelA and $\mathrm{P} c e l B$ ) was observed when the half or entire predicted CelR regulatory site was removed, in both CM17 (M17+0.5\% cellobiose) and GM17 $(\mathrm{M} 17+0.5 \%$ glucose $)$ medium. However, when the celA and $c e l B$ promoters were truncated only a few base pairs upstream of the predicted regulatory site, expression was similar to that of the full-length promoters (Fig. 3). Therefore, these data suggest that the putative predicted regulatory site is functional and acts as a CelR regulatory site. Moreover, since this predicted site is found to be highly conserved in other streptococci, it is most likely also functional as a CelR regulatory site in these species.

\section{DISCUSSION}

In this study, the expression of the cel locus of $S$. pneumoniae, which is important for growth on cellobiose, was shown to be activated by cellobiose and repressed by glucose via the transcriptional activator CelR. This regulatory effect depends on a $22 \mathrm{bp}$ palindromic sequence present in the two promoters, $\mathrm{P} c e l A$ and $\mathrm{PcelB}$, that drive expression of the cel locus.

Expression of the appropriate genes necessary to make efficient use of the available energy sources is of utmost importance for the fitness of pathogenic bacteria in a fluctuating environment. In low-G $+\mathrm{C}$ Gram-positive bacteria, including $S$. pneumoniae, carbon catabolite protein A (CcpA) is mainly responsible for the regulation of sugar metabolic genes in response to the available carbon source(s), which ensures their optimal use (Lulko et al., 2007; Zomer et al., 2007; S. M. Carvalho and others, unpublished). In this study, we observed that CcpA plays no role in the regulation of the cel locus in S. pneumoniae, despite the presence of a putative CRE site in the promoter of PcelA. Also, there was no effect on the expression of the 
(a)

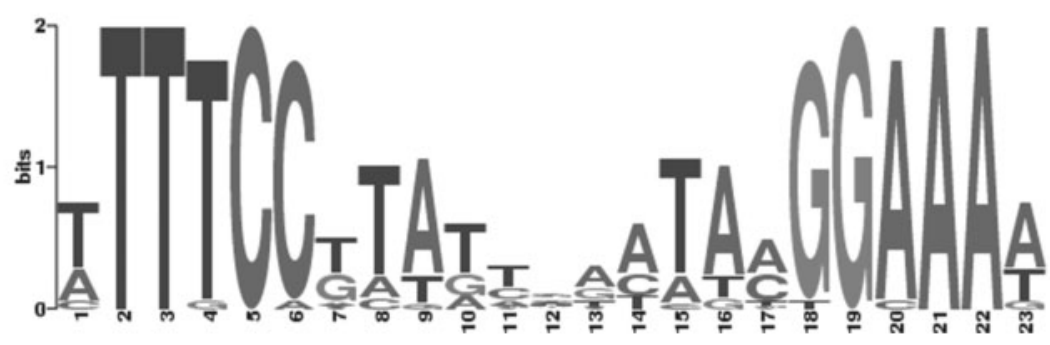

(b)

1-- TTTCCATTAAAAATTACTTCTTTCCTTAA CAATAAGGAAAAAGAATGCGTTTTTATCTTTTGGTTTAA 2-- ATTGAAGTATAAATAATTTTTTCCATAT CATTTAGGAAAATGAGGGAATTGTCATTGGAAACGCTTT

3-- TAGAATAAAATAAAAGTCTTTTTCCGTTT CGATATGGAAAATCTTGCTATTCAAAACAGCGCTTTCTT 4-- AAGTAGCTTCTTAGAAACCATTTCCGTTT CGATACGGAAAAAGCTCAAATGGAGATTGATGGCGTTTT 5-- AGGCTGCCCTAAGATGAGAATTCCTTAGTCTTTAAGGAAATTTTTCTATTTTAATTTTGAAATTGTT 6-- TAAAGAGGCTGTGTAAGGACTTTCCGTTTTCAATACGGAAAAACTGTGCGTTTACATTTTGTAAGCGCT 7-- AGAAATAAGAAATCGAGAAATTTCCTTAGTCACTAAGGAAATTTTTATATGGTCTATTGTGAAATCGC 8-- TAAAAATAAATAGATAATTTGCCGTAg TGCTAAgGAAAAAGTCTCTTTTGAAGTTGTAAAGGTTTT 9-- ATAAATAAAGATGTCAAAAATTTCCTAAT AACTTAGGAAATTTTTTCTATTCTCGTTTTTGTAACCGC 10-- TTCCGCACTTGGTGAAGTATTTTCCTTAT CAAAGAGGAAAAAGAGTACATTTACTTTATGTAAGCGTT 11-- TCAAGCTTTAAATCACTTATTTTCCGTGA TGATAAGGAAAAAACAGATTTTTTAAAGCGTTTACATCA 12-- ATTGCCCCATCATAGATTCTTTTCACAAT CAATGCGGAAAATGATGGTCACCTTTTTCAGGAAATATT 13-- GAATGATCATGTTGTCTTAATTTCCGTTG TGATACGGAAAAAGAGGGTCTGTGTATCCGTTTACAATA WTTTCCKTWTTCRATAAGGAAAA

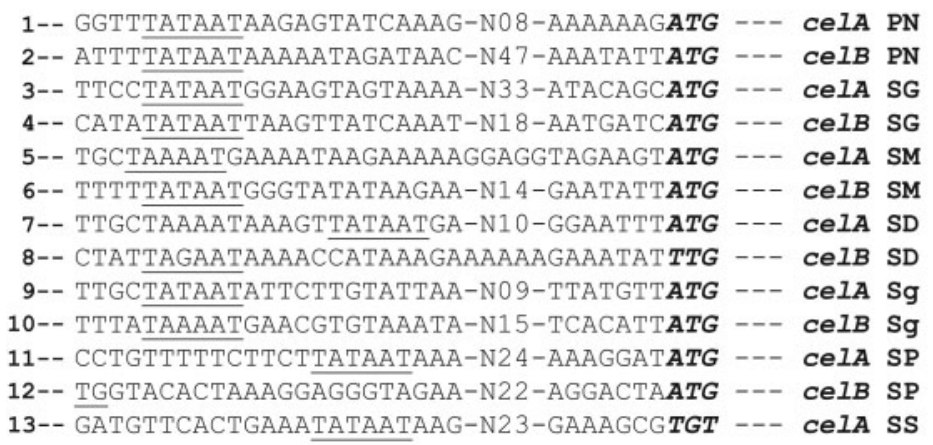

Fig. 2. Identification of a CelR regulatory site in different streptococci. (a) Weight matrix of the identified CelR regulatory site in $\mathrm{Pcel} A$ and PcelB from different streptococci. (b) Position of a CelR regulatory site in PcelA and PcelB of different streptococci. PN, S. pneumoniae; SG, S. gordonii; SM, S. mutans; SD, S. dysgalactiae; Sg, S. gallolyticus; SP, S. pyogenes; SS, S. suis. Core promoter sequences are underlined, translational start sites are in bold italic type and putative CelR regulatory sites are in bold type.

cel locus in a recent transcriptome-wide analysis of a $\operatorname{ccp} A$ deletion mutant in glucose and galactose (S. M. Carvalho and others, unpublished). Similarly, deletion of $\operatorname{ccp} A$ has no impact on the regulation of the cel locus in S. mutans (Zeng \& Burne, 2009). This means that most probably the CRE box present in PcelA is not functional.

A previous study showed that TCS08 activates the cel locus in strain R6 (McKessar \& Hakenbeck, 2007). We did not find an effect of a deletion of $h k 08$ on the expression of $\mathrm{P}$ celA and PcelB in strain D39. This means that TCS08 probably regulates the cel locus in response to a different stimulus than the sugar source in strain D39. Thus, it is likely that CelR is the only regulator involved in the regulation of the cel locus in response to the sugar source in strain D39. In addition, our bioinformatics analysis showed that $50 \%$ of the strains available at the KEGG website do not contain genes homologous to the cel locus, implying that the role of TCS08 lies outside regulation of the cel locus in these strains. The absence of the cel locus in these strains also suggests the presence of an alternative cellobiose utilization system, which, as determined by BLAST analyses, could be SPD_1830-1833, encoding a cellobiose-specific PTS. This second cellobiose system is highly conserved in all the strains 
(a)

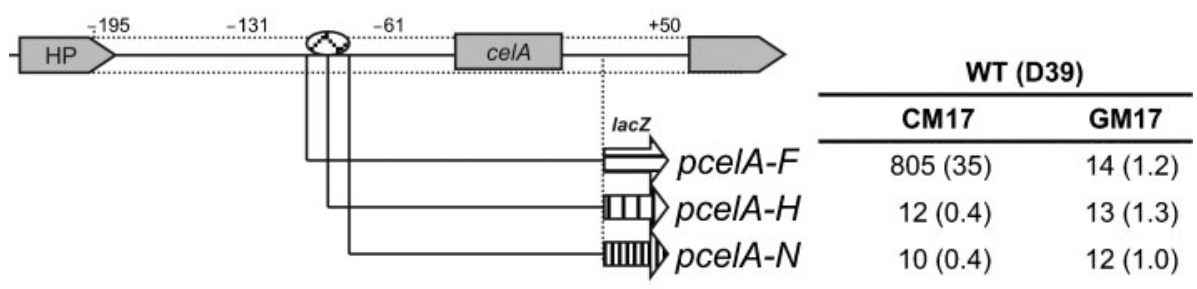

(b)

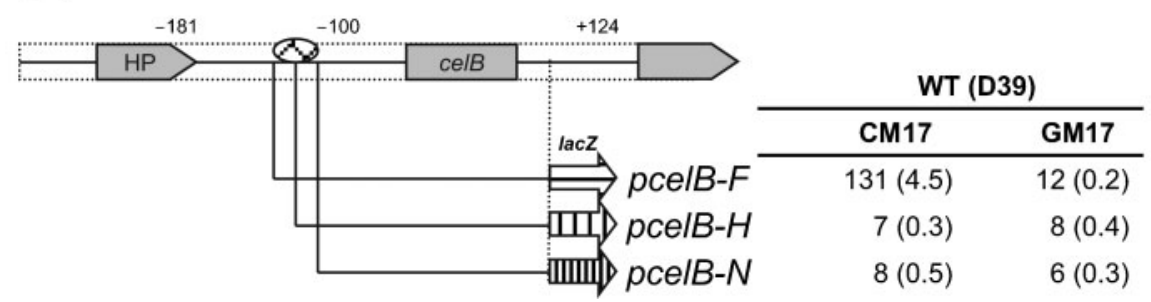

Fig. 3. Deletion analysis of $\mathrm{Pcel} A$ and $\mathrm{Pce} / B$. Schematic diagrams of the $\mathrm{Pcel} A(\mathrm{a})$ and $\mathrm{Pce} / B(\mathrm{~b})$ truncations are shown. Ovals indicate the position of the CelR regulatory site. To the right of each diagram are shown the specific $\beta$-galactosidase activities (Miller units) of the promoter truncations in D39 wild-type in GM1 7 (M17+0.5\% glucose) and CM1 7 (M17+0.5\% cellobiose) medium. Standard deviations of three independent measurements are given in parentheses.

available on the KEGG website. Therefore, it will be interesting to determine the role of this system in cellobiose metabolism in future studies.

Bioinformatic analysis revealed that CelR has two PTS regulation domains (PRDs), a helix-turn-helix (HTH) and EIIA and EIIB domains. Usually, transcriptional regulators with PRDs require phosphorylation on conserved histidine residues by one of the PTS components involved in the metabolism of a certain carbohydrate (van Tilbeurgh \& Declerck, 2001). Recently, the role of CelR $\left(\mathrm{CelR}^{\mathrm{SM}}\right)$ was established in the regulation of the cel locus of $S$. mutans (Zeng \& Burne, 2009). CelR ${ }^{\mathrm{SM}}$-dependent regulation of the cel locus in the presence of cellobiose required the phosphorylation of CelR ${ }^{S M}$ by CelD. Deletion of the second or both PRDs present in the CelR ${ }^{S M}$ protein led to the total inactivation of expression of the cel locus (Zeng \& Burne, 2009). It was further shown that phosphorylation at H226, $\mathrm{H} 332$ and $\mathrm{H} 576$ is required for the activation of $\mathrm{CelR}^{\mathrm{SM}}$ in the presence of cellobiose, while phosphorylation of $\mathrm{H} 284$ and $\mathrm{H} 391$ leads to inhibition of the $\mathrm{CelR}^{\mathrm{SM}}$-dependent activation in $S$. mutans in the presence of glucose. Interestingly, alignment of $\mathrm{CelR}^{\mathrm{SM}}$ showed that all the above-mentioned histidine residues are conserved in the CelR protein (data not shown). The presence of these conserved histidines suggests that the activity of CelR is determined in the same manner as $\mathrm{CelR}^{\mathrm{SM}}$. This is supported by a previous study, showing that S. pneumoniae R6 lacking celD is unable to grow on cellobiose (McKessar \& Hakenbeck, 2007).

Previous signature-tagged mutagenesis (STM) screens highlighted the importance of CelR for the virulence of
S. pneumoniae (Hava \& Camilli, 2002; Lau et al., 2001). So far, however, a possible role in virulence of the other genes in the cel locus has never been investigated. Since CelR is dedicated exclusively to the regulation of the cel locus, it is likely that the attenuated virulence of a celR mutant is caused by deactivation of the expression of the genes present in the cel locus. However, since several pneumococcal strains, such as AP200, ATCC 700669-23F, G54-19F, Hungary-19A, JJA, P1031 and TCH8431/19A, lack the cel locus, the contribution of the cel locus to virulence seems to be strain-specific.

As cellobiose is unlikely to be present in the human host, the natural substrate(s) for the PTS encoded by the cel locus remain(s) to be determined. It is known that $S$. pneumoniae cleaves host glucosaminoglycans by the activity of many glucosidases, thereby releasing usable carbon sources (King, 2010). On the basis of their disaccharide units and linkage pattern, glycosaminoglycans are classified into three structural groups (Li et al., 2000; Scott \& Heatley, 1999): (i) the cellobiose type (HA: hyaluronic acid or hyaluronate); (ii) the polylactose type (chondroitin and keratan sulfates); and (iii) the polymaltose type (heparan sulfate) (Li et al., 2000). In a previous study (McKessar \& Hakenbeck, 2007), it was shown that deletion of $c e l R$ has no effect on the growth of strain R6 in the presence of HA. This suggests that HA is not metabolized via the Cel system and that HA is not involved in the regulation of the cel locus. Therefore, it could be that $\beta$-linked disaccharides derived from degradation of other host glycosaminoglycans serve as substrates for the Cel system. 


\section{ACKNOWLEDGEMENTS}

Funds for S. S. were made available under the HEC-NUFFIC overseas scholarship programme by the Higher Education Commission (HEC) Pakistan. T. G. K. is supported by the EU-funded Pneumopath project HEALTH-F3-2009-222983.

\section{REFERENCES}

Bailey, T. L. \& Elkan, C. (1994). Fitting a mixture model by expectation maximization to discover motifs in biopolymers. Proc Int Conf Intell Syst Mol Biol 2, 28-36.

Bogaert, D., De Groot, R. \& Hermans, P. W. (2004). Streptococcus pneumoniae colonisation: the key to pneumococcal disease. Lancet Infect Dis 4, 144-154.

Brown, S. A., Palmer, K. L. \& Whiteley, M. (2008). Revisiting the host as a growth medium. Nat Rev Microbiol 6, 657-666.

Deutscher, J., Francke, C. \& Postma, P. W. (2006). How phosphotransferase system-related protein phosphorylation regulates carbohydrate metabolism in bacteria. Microbiol Mol Biol Rev 70, 939-1031.

Halfmann, A., Hakenbeck, R. \& Brückner, R. (2007). A new integrative reporter plasmid for Streptococcus pneumoniae. FEMS Microbiol Lett 268, 217-224.

Hava, D. L. \& Camilli, A. (2002). Large-scale identification of serotype 4 Streptococcus pneumoniae virulence factors. Mol Microbiol 45, 1389-1406.

Kadioglu, A., Weiser, J. N., Paton, J. C. \& Andrew, P. W. (2008). The role of Streptococcus pneumoniae virulence factors in host respiratory colonization and disease. Nat Rev Microbiol 6, 288-301.

Keyhani, N. O. \& Roseman, S. (1997). Wild-type Escherichia coli grows on the chitin disaccharide, $N, N^{\prime}$-diacetylchitobiose, by expressing the cel operon. Proc Natl Acad Sci U S A 94, 14367-14371.

Kiliç, A. O., Tao, L., Zhang, Y., Lei, Y., Khammanivong, A. \& Herzberg, M. C. (2004). Involvement of Streptococcus gordonii beta-glucoside metabolism systems in adhesion, biofilm formation, and in vivo gene expression. J Bacteriol 186, 4246-4253.

King, S. J. (2010). Pneumococcal modification of host sugars: a major contributor to colonization of the human airway? Mol Oral Microbiol $25,15-24$.

Kloosterman, T. G., Bijlsma, J. J. E., Kok, J. \& Kuipers, O. P. (2006a). To have neighbour's fare: extending the molecular toolbox for Streptococcus pneumoniae. Microbiology 152, 351-359.

Kloosterman, T. G., Hendriksen, W. T., Bijlsma, J. J., Bootsma, H. J., van Hijum, S. A., Kok, J., Hermans, P. W. \& Kuipers, O. P. (2006b). Regulation of glutamine and glutamate metabolism by GlnR and GlnA in Streptococcus pneumoniae. J Biol Chem 281, 25097-25109.

Lanie, J. A., Ng, W. L., Kazmierczak, K. M., Andrzejewski, T. M., Davidsen, T. M., Wayne, K. J., Tettelin, H., Glass, J. I. \& Winkler, M. E. (2007). Genome sequence of Avery's virulent serotype 2 strain D39 of Streptococcus pneumoniae and comparison with that of unencapsulated laboratory strain R6. J Bacteriol 189, 38-51.

Lau, G. W., Haataja, S., Lonetto, M., Kensit, S. E., Marra, A., Bryant, A. P., McDevitt, D., Morrison, D. A. \& Holden, D. W. (2001). A functional genomic analysis of type 3 Streptococcus pneumoniae virulence. Mol Microbiol 40, 555-571.

Leenhouts, K., Buist, G., Bolhuis, A., ten Berge, A., Kiel, J., Mierau, I., Dabrowska, M., Venema, G. \& Kok, J. (1996). A general system for generating unlabelled gene replacements in bacterial chromosomes. Mol Gen Genet 253, 217-224.
Li, S., Kelly, S. J., Lamani, E., Ferraroni, M. \& Jedrzejas, M. J. (2000). Structural basis of hyaluronan degradation by Streptococcus pneumoniae hyaluronate lyase. EMBO J 19, 1228-1240.

Long, A. D., Mangalam, H. J., Chan, B. Y., Tolleri, L., Hatfield, G. W. \& Baldi, P. (2001). Improved statistical inference from DNA microarray data using analysis of variance and a Bayesian statistical framework. Analysis of global gene expression in Escherichia coli K12. J Biol Chem 276, 19937-19944.

Lulko, A. T., Buist, G., Kok, J. \& Kuipers, O. P. (2007). Transcriptome analysis of temporal regulation of carbon metabolism by CcpA in Bacillus subtilis reveals additional target genes. J Mol Microbiol Biotechnol 12, 82-95.

McKessar, S. J. \& Hakenbeck, R. (2007). The two-component regulatory system TCS08 is involved in cellobiose metabolism of Streptococcus pneumoniae R6. J Bacteriol 189, 1342-1350.

Mitchell, T. J. (2003). The pathogenesis of streptococcal infections: from tooth decay to meningitis. Nat Rev Microbiol 1, 219-230.

Obaro, S. \& Adegbola, R. (2002). The pneumococcus: carriage, disease and conjugate vaccines. J Med Microbiol 51, 98-104.

Old, L. A., Lowes, S. \& Russell, R. R. (2006). Genomic variation in Streptococcus mutans: deletions affecting the multiple pathways of $\beta$ glucoside metabolism. Oral Microbiol Immunol 21, 21-27.

Scott, J. E. \& Heatley, F. (1999). Hyaluronan forms specific stable tertiary structures in aqueous solution: a ${ }^{13} \mathrm{C}$ NMR study. Proc Natl Acad Sci U S A 96, 4850-4855.

Shafeeq, S., Kloosterman, T. G. \& Kuipers, O. P. (2011a). Transcriptional response of Streptococcus pneumoniae to $\mathrm{Zn}^{2+}$ limitation and the repressor/activator function of AdcR. Metallomics 3, 609-618.

Shafeeq, S., Yesilkaya, H., Kloosterman, T. G., Narayanan, G., Andrew, P. W., Kuipers, O. P. \& Morrissey, J. A. (2011b). The cop operon is required for copper homeostasis and contributes to virulence in Streptococcus pneumoniae. Mol Microbiol [Epub ahead of print]. doi:10.1016/S1473-3099(04)00938-7.

Shelburne, S. A., Davenport, M. T., Keith, D. B. \& Musser, J. M. (2008). The role of complex carbohydrate catabolism in the pathogenesis of invasive streptococci. Trends Microbiol 16, 318-325.

Song, X. M., Connor, W., Hokamp, K., Babiuk, L. A. \& Potter, A. A. (2009). The growth phase-dependent regulation of the pilus locus genes by two-component system TCS08 in Streptococcus pneumoniae. Microb Pathog 46, 28-35.

Terzaghi, B. E. \& Sandine, W. E. (1975). Improved medium for lactic streptococci and their bacteriophages. Appl Microbiol 29, 807-813.

Throup, J. P., Koretke, K. K., Bryant, A. P., Ingraham, K. A., Chalker, A. F., Ge, Y., Marra, A., Wallis, N. G., Brown, J. R. \& other authors (2000). A genomic analysis of two-component signal transduction in Streptococcus pneumoniae. Mol Microbiol 35, 566-576.

van Hijum, S. A. F. T., García de la Nava, J., Trelles, O., Kok, J. \& Kuipers, O. P. (2003). MicroPreP: a cDNA microarray data preprocessing framework. Appl Bioinformatics 2, 241-244.

van Tilbeurgh, H. \& Declerck, N. (2001). Structural insights into the regulation of bacterial signalling proteins containing PRDs. Curr Opin Struct Biol 11, 685-693.

Zeng, L. \& Burne, R. A. (2009). Transcriptional regulation of the cellobiose operon of Streptococcus mutans. J Bacteriol 191, 2153-2162.

Zomer, A. L., Buist, G., Larsen, R., Kok, J. \& Kuipers, O. P. (2007). Time-resolved determination of the CcpA regulon of Lactococcus lactis subsp. cremoris MG1363. J Bacteriol 189, 1366-1381.

Edited by: T. J. Mitchell 